\title{
Experimental Vehicle Localization by Bounded-Error State Estimation Using Interval Analysis
}

\author{
Emmanuel Seignez*, Michel Kieffer**, Alain Lambert*, Eric Walter** and Thierry Maurin* \\ * Institut d'Électronique Fondamentale \\ CNRS - Université de Paris-Sud, 11 \\ Bat. 220, 91405 Orsay, France \\ name@ief.u-psud.fr \\ ** Laboratoire des Signaux et Systèmes \\ CNRS - Supélec - Université de Paris-Sud, 11 \\ Plateau du Moulon, 91192 Gif-sur-Yvette, France \\ name@1ss.supelec.fr
}

\begin{abstract}
Estimating the configuration of a vehicle is crucial for navigation. The most classical approaches are Kalman filtering and Bayesian localization, often implemented via particle filtering. This paper reports on-going experimentation with an attractive alternative approach recently developed and based on interval analysis. Contrary to classical Extended Kalman Filtering, this approach allows global localization, and contrary to Bayesian localization it provides guaranteed results in the sense that a set is computed that contains all of the configurations that are consistent with the data and hypotheses. The approach is particularly robust to outliers.

Index Terms-Bounded-error estimation, interval analysis, outliers, robust localization.
\end{abstract}

\section{INTRODUCTION}

Estimating the configuration (position and orientation) of a vehicle in a known environment is a crucial problem in navigation. Most of the work published uses the same types of proprioceptive and exteroceptive sensors [1], namely odometers for the former and ultrasonic or laser telemeters for the latter. Localization usually alternates a prediction phase and a correction (estimation) phase. Prediction integrates the odometric data according to a procedure that is more or less always the same, contrary to estimation which may be performed in very different ways. The most commonly used methods are based on Kalman filtering [2], [3] and Bayesian localization, using either a probability grid [4], or particle filtering [5].

Kalman filtering [6] does not require a great amount neither of computing power nor of memory but, in return, cannot perform global localization and can only track one configuration of the vehicle. Moreover, it diverges very swiftly in presence of erroneous data (outliers) even using methods developed to overcome some of its limitations such as reported in [7]. Kalman filtering is therefore not well adapted to situations where outliers are unavoidable.

As regards Bayesian localization techniques, they require more computing power but provide more reliable estimated configurations in complex, dynamic or badly mapped environments [4]. They have been reigning for the last few years and much work is still going on to improve them, especially Monte Carlo localization [8]. These methods evaluate the probability for a vehicle to be in a given region of a mapped environment but nothing ensures that the vehicle is indeed in the configuration with the highest probability.

Alternative and less known methods have been developed for localization, such as those based on animat research [9] or bounded-error state estimation [10], which is studied here.

In bounded-error state estimation, all model and measurement errors are assumed to be bounded, with known bounds. Then, at each time instant, the bounded-error recursive state estimator provides a set containing all possible configurations of the vehicle given the measurements and noise bounds. The methodology has now been in existence for quite some time but had so far proved its feasibility in simulations [10]. The goal of this paper is to demonstrate that it can be made operational on an actual vehicle.

In the following sections, we first briefly describe the vehicle architecture and the models used. Next, boundederror localization is presented and some experimental results based on this technique are reported.

\section{EXPERIMENTAL SET-UP}

\section{A. The vehicle}

To facilitate testing, we designed a generic mobile robot called Minitruck, which had to be light and small.

As some localization algorithms, like bounded-error localization considered here, require a lot of computing power (not easily implementable on board), a client-server architecture with a powerful distant workstation was chosen. This architecture also makes it possible to deport the task of path planning and control to a distant computer or operator.

Minitruck is built on the mechanical base of a vehicle model with a $50 \mathrm{~cm}$ length and $25 \mathrm{~cm}$ width. Its motion is measured by two incremental coders mounted on each rear wheel which give a millimeter-length accuracy.

The exteroceptive sensors, a ring of ten ultrasonic modules (sonars), are located as indicated on Fig. 1. Up to sixteen sensors can be accommodated. Experimentation in various conditions showed that the sonars are blind below $3 \mathrm{~cm}$ and that their maximum sensing distance is $6 \mathrm{~m}$. 


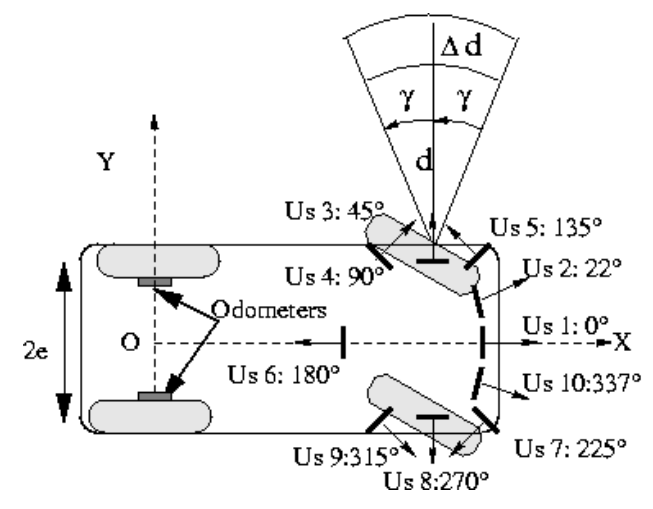

Fig. 1. Location of the sensors and uncertainty of the sonar measurements

Their relative uncertainty $\Delta d$ is taken as $0.03 \times d$ and their opening angle $2 \gamma$ is taken as $45^{\circ}$.

\section{B. Map, odometric and sensor models of Minitruck}

A 2D world map $\mathcal{M}$ of the environment is considered. Walls and obstacles are described by polygons.

The vehicle configuration in the global frame is denoted by $\mathbf{x}=\left(x_{c}, y_{c}, \theta_{c}\right)^{\mathrm{T}}$ where $\left(x_{c}, y_{c}\right)$ are the coordinates of the middle $\mathrm{O}$ of the segment joining the two steering wheels and $\theta_{c}$ specifies the orientation of a local frame attached to the vehicle with respect to the global frame. A nonlinear discrete-time state-space model is considered to describe the evolution of the configuration $\mathbf{x}_{k}$ of Minitruck

$$
\mathbf{x}_{k+1}=\mathbf{g}\left(\mathbf{x}_{k}, \mathbf{u}_{k}, \mathbf{v}_{k}\right)
$$

where $\mathbf{u}_{k}$ is a known two-dimensional control vector, assumed constant between the times indexed by $k$ and $k+1$ and $\mathbf{v}_{k}$ is an unknown state perturbation vector that accounts for the uncertain description of reality by this model.

The vehicle model is a discrete approximation of a continuous time model with integration by finite differences. The control inputs are the angle of the two front wheels in the vehicle frame and the mean speed of the rear wheels. The motion $\omega_{r}$ and $\omega_{\ell}$ of the right and left rear wheels are measured by incremental coders during each time period $\delta t$. These motions can be translated into the incremental longitudinal motion $\delta s$ and rotational motion $\delta \theta$ as

$$
\delta s=\frac{\delta r+\delta \ell}{2} \text { and } \delta \theta=\frac{\delta r-\delta \ell}{2(e+\Delta e)},
$$

where $\delta r=(R+\Delta R) \omega_{r}$ and $\delta \ell=(R+\Delta R) \omega_{\ell}$ are the displacements of the right and left rear wheels, $R$ is the radius of a rear wheel and $2 e$ is the distance between these two wheels. The errors $\Delta R$ on $R$ and $\Delta e$ on the distance between wheels are the main causes of discrepancy between the car and model behaviors so we shall assume that $\mathbf{v}_{k}=(\Delta R, \Delta e)^{\mathrm{T}}$.

To cancel the effect of the error between the desired control input and its actual value when applied to the vehicle, the input is taken as the motion of the rear wheels $\mathbf{u}_{k}=\left(\omega_{r}, \omega_{\ell}\right)^{\mathrm{T}}$ in the state equations describing the motion of the vehicle, which can thus be described as follows

$$
\begin{aligned}
\mathbf{x}_{k+1}= & \mathbf{g}\left(\mathbf{x}_{k}, \mathbf{u}_{k}, \mathbf{v}_{k}\right) \\
= & \left(\begin{array}{c}
x_{k}+\delta s \cdot \cos \left(\theta_{k}+\delta \theta / 2\right) \\
y_{k}+\delta s \cdot \sin \left(\theta_{k}+\delta \theta / 2\right) \\
\theta_{k}+\delta \theta
\end{array}\right)
\end{aligned}
$$

To implement the estimation steps of all localization algorithms, a model of the measurement process has to be built. It is assumed that the wave emitted by a sonar propagates inside a cone with aperture $2 \gamma$, and that the distance reported by a given sonar corresponds to the closest charted detectable obstacle at least partly located in the emission cone [10], [11]. For any given configuration $\mathbf{x}$, it is then possible to compute the $n_{\mathrm{S}}$ expected distances $\mathbf{y}_{\mathrm{m}}(\mathbf{x}), n_{\mathrm{s}}$ being the number of ultrasonic sensors, which should match the $n_{\mathrm{s}}$ actual distances $\mathbf{y}$. The nonlinear measurement equation is

$$
\mathbf{y}_{k}=\mathbf{y}_{\mathrm{m}}\left(\mathbf{x}_{k}\right)+\mathbf{w}_{k}
$$

where $\mathbf{w}_{k}$ is the measurement noise vector.

\section{BOUNDED-ERROR LOCALIZATION USING INTERVAL ANALYSIS}

\section{A. Basic concepts of interval analysis}

Interval analysis [12]-[14] allows computation with intervals $[x]=[\underline{x}, \bar{x}]$ and vector intervals or boxes $[\mathbf{x}]=$ $[\underline{\mathbf{x}}, \overline{\mathbf{x}}]$. Arithmetical operations $(+,-, *$ and $/)$ and standard mathematical functions readily extend to intervals. For example, $[2,4]+[4,5]=[6,9]$ and $\exp ([0,1])=[1, e]$. The width of an interval $w([x])=\bar{x}-\underline{x}$ or of a box $w([\mathbf{x}])=\max _{i=1 \ldots n} w\left(\left[x_{i}\right]\right)$ is used to quantify its size.

The notion of inclusion function is one of the most important tool provided by interval analysis. An inclusion function $[f]($.$) of a given function f($.$) defined over \mathcal{D} \subset$ $\mathbb{R}$ is an interval-valued function that satisfies

$$
\forall[x] \subset \mathcal{D}, f([x]) \subset[f]([x]),
$$

where $f([x])$ denotes the set of all values taken by $f($. over $[x]$.

The simplest way to obtain an inclusion function is to replace all real variables by interval ones and all real-valued operators or elementary functions by their interval counterparts. The natural inclusion function is then obtained. For example, the natural inclusion function for

$$
f(x)=x^{3}-\exp (x)+1
$$

is

$$
[f]([x])=[x]^{3}-\exp ([x])+1 .
$$

For more details about inclusion functions, see, e.g., [14].

Outer-approximation of sets may be achieved by union of non-overlapping boxes or subpavings. Subpavings combined with direct image evaluation and inverse image evaluation algorithms are the building stones of the boundederror state estimation algorithm which will now be briefly recalled. 


\section{B. Overview of bounded-error state estimation}

All model and measurement errors (from odometers and telemeters) are assumed bounded with known bounds. At each time instant $k$, the bounded-error recursive state estimator will provide a set $S_{k}$ guaranteed to contain all configurations of the vehicle that are consistent with the information $\mathcal{I}_{k}$ available at time $k$ (map, measurements, error bounds), provided that the hypotheses on the error bounds are not violated. More precisely $S_{k}$ will be outerapproximated by a subpaving [14]. Since all computed subpavings may consist of many disconnected components, multiple-configuration tracking which may be necessary because of initial ambiguities due to local symmetries in the environment, can be achieved using exactly the same tools as single-configuration tracking. A consequence is that no precise initial localization is required. Moreover, the technique is easily tuned to become particularly robust to outliers, which cannot be avoided when considering sonar measurements, as mentioned in Section III-D.

Between time $k$ and time $k+1$, the control vector $\mathbf{u}_{k}$ is assumed available as well as a box $\left[\mathbf{v}_{k}\right]$ guaranteed to contain the perturbation vector $\mathbf{v}_{k}$ in (1). At time $k+1$, a vector $\mathbf{y}_{k}$ of $n_{\mathrm{s}}$ distance measurements is assumed available, as well as a box $\left[\mathbf{w}_{k}\right]$ guaranteed to contain the vector $\mathbf{w}_{k}$ of measurement noise in (4).

The problem to be treated is then to estimate $\mathbf{x}_{k}$ in real time from the information available up to time $k$, i.e.,

$$
\mathcal{I}_{k}=\left\{S_{0},\left\{\mathbf{u}_{i}, \mathbf{y}_{i},\left[\mathbf{v}_{i}\right],\left[\mathbf{w}_{i}\right]\right\}_{i=0}^{k}\right\},
$$

where $S_{0}$ is a (possibly very large) set guaranteed to contain the initial vehicle configuration.

At each discrete time instant $k$, an outer approximation of the set $S_{k}$ of all values of the state that are consistent with $\mathcal{I}_{k}$ will be computed. The procedure is recursive and alternates a prediction phase, during which an outer approximation $\bar{S}_{k+1 / k}$ of the set $S_{k+1 / k}=\mathbf{g}\left(S_{k}, \mathbf{u}_{k},\left[\mathbf{v}_{k}\right]\right)$ of all $\mathbf{x}_{k+1}$ that are consistent with $\mathcal{I}_{k}$ is built using (1), and a correction phase, during which $\mathcal{I}_{k+1}$, which includes the new data vector $\mathbf{y}_{k+1}$, is taken into account to update $\bar{S}_{k+1 / k}$ into $\bar{S}_{k+1}$

During prediction, $\bar{S}_{k+1 / k}$ is computed as an outer approximation of the set $\mathbf{g}\left(\bar{S}_{k}, \mathbf{u}_{k},\left[\mathbf{v}_{k}\right]\right)$, which is guaranteed to contain $\mathbf{g}\left(S_{k}, \mathbf{u}_{k},\left[\mathbf{v}_{k}\right]\right)$, as $S_{k} \subset \bar{S}_{k}$. During correction, $\bar{S}_{k+1}$ is computed as an outer approximation of

$$
\begin{aligned}
S_{k+1} & =\left\{\mathbf{x} \in \bar{S}_{k+1 / k} \mid \mathbf{y}_{\mathrm{m}}(\mathbf{x}) \in\left(\mathbf{y}_{k}-\left[\mathbf{w}_{k}\right]\right)\right\} \\
& =\bar{S}_{k+1 / k} \cap \mathbf{y}_{\mathrm{m}}^{-1}\left(\left[\mathbf{y}_{k}\right]\right)
\end{aligned}
$$

with $\left[\mathbf{y}_{k}\right]=\mathbf{y}_{k}-\left[\mathbf{w}_{k}\right]$

Prediction thus involves the computation of the direct image of a set by the function $\mathrm{g}$ while correction involves the computation of the image of a set by the inverse of the function $\mathbf{y}_{\mathrm{m}}(\mathbf{x})$. All these steps may be implemented in an approximate but guaranteed way by using interval analysis. The resulting state estimator is a bounded-error nonlinear counterpart to Kalman filtering [14].

Remark 1: Correlation between components of $\mathbf{v}_{k}$ and between components of $\mathbf{w}_{k}$ could readily be taken into account by bounding state perturbation and measurement noise vectors in subpavings.

\section{Prediction step}

The direct image evaluation algorithm IMAGESP [14] can be put at work during prediction to evaluate an outer approximation $\bar{S}_{k+1 / k}$ of the set $\mathbf{g}\left(\bar{S}_{k}, \mathbf{u}_{k},\left[\mathbf{v}_{k}\right]\right)$. This is performed in three steps, provided that an inclusion function for $\mathbf{g}$ is available.

First, the subpaving $\bar{S}_{k}$ is minced, i.e., all boxes of $\bar{S}_{k}$ are bisected until their width is lower than some specified precision parameter $\varepsilon_{\mathrm{I}}$.

The image of all these boxes is then evaluated using the inclusion function for $\mathrm{g}$. According to (5), the union of all these images is guaranteed to contain $\mathbf{g}\left(\bar{S}_{k}, \mathbf{u}_{k},\left[\mathbf{v}_{k}\right]\right.$.

Finally, all the images are merged to get a new subpaving $\bar{S}_{k+1 / k}$

The mincing allows a better estimation of the image to be obtained. When $\varepsilon_{\mathrm{I}}$ gets smaller, an image subpaving $\bar{S}_{k+1 / k}$ gets closer (in the sense of the Hausdorf distance) to $S_{k+1 / k}$. The price to be paid is an increased computing time.

\section{Correction step}

The correction step is implemented using the SIVIA algorithm [14], which eliminates from $\bar{S}_{k+1 / k}$ all configuration boxes that can be proved to be incompatible with the measurements and noise bounds at time $k+1$ to get the subpaving $\bar{S}_{k+1}$. In this case, an inclusion function $\left[\mathbf{y}_{\mathrm{m}}\right]$ (.) for $\mathbf{y}_{\mathrm{m}}($.$) is required. To all boxes [\mathbf{x}]$ of $\bar{S}_{k+1 / k}$, the following tests are applied.

If $\left[\mathbf{y}_{\mathrm{m}}\right]([\mathbf{x}]) \subset\left[\mathbf{y}_{k}\right]$, then according to $(5)$, for all configurations $\mathbf{x} \in[\mathbf{x}], \mathbf{y}_{\mathrm{m}}(\mathbf{x}) \in\left[\mathbf{y}_{k}\right]$. The vector $\mathbf{x}$ is thus consistent with the measurements and noise bounds and $[\mathbf{x}]$ can be stored in $\bar{S}_{k+1}$.

If $\left[\mathbf{y}_{\mathrm{m}}\right]([\mathbf{x}]) \cap\left[\mathbf{y}_{k}\right]=\emptyset$, then, again according to $(5)$, for all $\mathbf{x} \in[\mathbf{x}], \mathbf{y}_{\mathrm{m}}(\mathbf{x}) \notin\left[\mathbf{y}_{k}\right]$. Therefore, there is no $\mathbf{x}$ in $[\mathbf{x}]$ that is consistent with the measurements and noise bounds and thus $[\mathbf{x}]$ does not belong to $\bar{S}_{k+1}$.

In all other cases, if $w([\mathbf{x}])$ is larger than some specified precision parameter $\varepsilon_{\mathrm{S}}$, it is bisected across its dimension of larger width to get two subboxes on which the same tests are applied again. If $w([\mathbf{x}])<\varepsilon_{\mathbf{S}},[\mathbf{x}]$ is stored in $\bar{S}_{k+1}$.

For the ultrasonic range measurements, sometimes more than $50 \%$ of the collected data may correspond to outliers. One of the major sources of outliers is multiple reflections. As a consequence, $\bar{S}_{k+1}$ may very frequently turn out to be empty. To solve this problem, a correction technique robust to outliers has to be used in place of the basic correction step.

Consider the interval consistency test for the $i$-th range measurement defined as follows

$$
\left[t_{i}\right]([\mathbf{x}])=\left\{\begin{array}{cl}
1 & \text { if }\left[y_{\mathrm{m}, i}\right]([\mathbf{x}]) \subset\left[y_{k, i}\right] \\
0 & \text { if }\left[y_{\mathrm{m}, i}\right]([\mathbf{x}]) \cap\left[y_{k, i}\right]=\emptyset \\
{[0,1]} & \text { otherwise }
\end{array}\right.
$$


with $\left[y_{\mathrm{m}, i}\right]([\mathbf{x}])$ and $\left[y_{k, i}\right]$ representing the $i$-th component of $\left[\mathbf{y}_{\mathbf{m}}\right]([\mathbf{x}])$ and $\left[\mathbf{y}_{k}\right]$ and the interval $[0,1]$ corresponding to an inconclusive test. Then, if $n_{\mathrm{s}}$ is the number of sonars,

$$
\begin{aligned}
& \sum_{i=1}^{n_{\mathrm{s}}}\left[t_{i}\right]([\mathbf{x}])=n_{\mathrm{s}} \Leftrightarrow\left[\mathbf{y}_{\mathrm{m}}\right]([\mathbf{x}]) \subset\left[\mathbf{y}_{k}\right] \\
& \sum_{i=1}^{n_{\mathrm{s}}}\left[t_{i}\right]([\mathbf{x}])<n_{\mathrm{s}} \Leftrightarrow\left[\mathbf{y}_{\mathrm{m}}\right]([\mathbf{x}]) \cap\left[\mathbf{y}_{k}\right]=\emptyset .
\end{aligned}
$$

More precisely, $\sum_{i=1}^{n_{\mathrm{s}}}\left[t_{i}\right]([\mathbf{x}]) \geqslant n$ implies that any $\mathbf{x} \in$ $[\mathbf{x}]$, is consistent with at least $n$ measurements among the $n_{\mathrm{s}}$ available.

The characterization of the set $\bar{S}_{k+1}$ of all configurations in $\bar{S}_{k+1 / k}$ consistent with all measurements and noise bounds can be relaxed to the characterization of the set $\bar{S}_{k+1}^{n}$ of all configurations in $\bar{S}_{k+1 / k}$ that are consistent with at least $n$ measurements. One possible implementation of the robust correction step will initialize $n$ at $n_{\mathrm{s}}$ and decrease it until a nonempty set $\bar{S}_{k+1}^{n}$ is obtained. To increase robustness, more outliers than strictly necessary may be tolerated to obtain a nonempty set. The price to be paid for this enhanced robustness is a degradation of the localization accuracy and an increase of the computational complexity when more outliers than strictly necessary are tolerated, as shown in the next section.

\section{E. Complexity}

This section provides a brief analysis of the evolution of (worst-case) complexity with the various factors of the localization technique considered here.

During the prediction step, the evaluation of the image of each box resulting from mincing is the most timeconsuming computation achieved by IMAGESP. It is linear with the number of boxes. This number depends on the volume of the subpaving $\bar{S}_{k}$ and on the parameter $\varepsilon_{\mathrm{I}}$. Since the dimension of the state vector is three, division of $\varepsilon_{\mathrm{I}}$ by 2 entails a multiplication of the number of boxes by $2^{3}$.

During the correction step, the most time-consuming part is the simulation of the ultrasonic sensors. If the map consists of $n_{\mathrm{w}}$ segments, for each of the $n_{\mathrm{s}}$ sensors at most $n_{\mathrm{w}}$ distances have to be computed. The complexity of the simulation part is thus linear in both $n_{\mathrm{s}}$ and $n_{\mathrm{w}}$. Setinversion with SIVIA is involved to obtain set estimates for the three parameters characterizing the configuration of Minitruck. The complexity of this technique is exponential in the number of parameters [15]. Here, three parameters only have to be estimated and the complexity remains tractable. When there are outliers, a procedure is repeated that is basically independent of the number of outliers, assuming no outliers, one outlier, two outliers, etc., so complexity is linear in the number of tolerated outliers. In summary, the overall complexity is linear in the number of outliers, in $n_{\mathrm{w}}$ and in $n_{\mathrm{s}}$.

Some computing times for actual experiments are provided in Section IV-B.

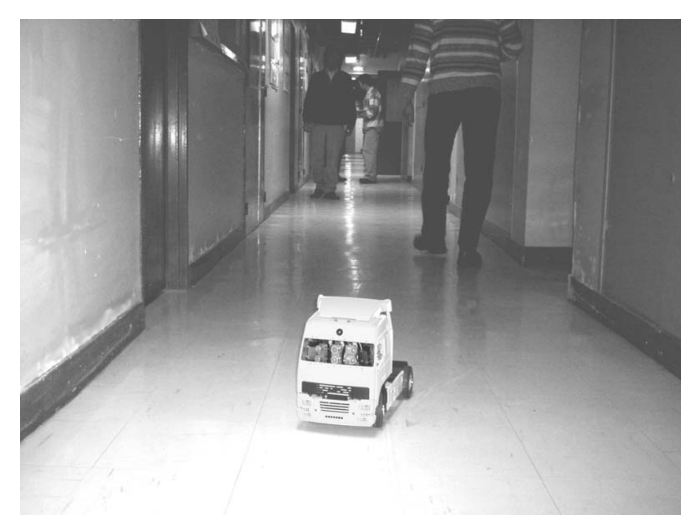

Fig. 2. Minitruck in its environment

Final configuration as predicted from odometric data

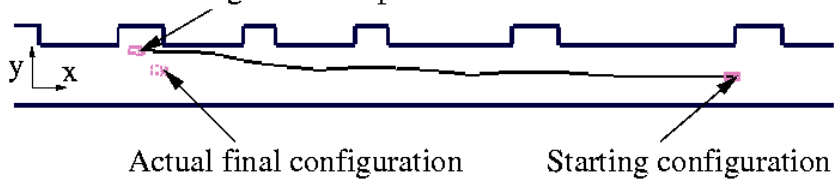

Fig. 3. Position of Minitruck as evaluated from odometric data alone

\section{EXPERIMENTAL RESULTS}

To illustrate the ability of the bounded-error localization technique to provide accurate estimates, even in presence of outliers, three examples are considered. The model uncertainties are taken as $\Delta R=0.05 R$ and $\Delta e=0.05 e$ and include both measurements errors and wheel slipping. For IMAGESP and SIVIA, the precision parameters $\varepsilon_{\mathrm{I}}$ and $\varepsilon_{\mathrm{S}}$ are both taken equal to $5 \mathrm{~cm}$.

\section{A. Tracking using bounded-error state estimation}

Fig. 3 represents the first experimental results, Minitruck moved within a corridor of our laboratory at a speed of $10 \mathrm{~cm} / \mathrm{s}$ (Fig. 2).

The curve in solid line, which represents the vehicle trajectory as provided by the odometers, deviates from the actual trajectory and crosses a wall. Such a localization error is due to an error in the initial configuration, to wheel slipping on the ground during the experiment and to inaccurate values of the distance between the rear wheels and of their diameter. On a twenty-meter course, the error on the arrival point is almost of two meters.

Sonar measurements were collected each second. Unfortunately, a large part of these data has to be considered as erroneous and does not correspond to any charted element. The presence of outliers is due not only to the uncertainty of the results returned by the sensors but also to foreign elements not represented on the map which were in the field of the sensors at the time of measurements, such as people walking in the corridor and uncharted elements.

Thus, the correction step should only consider the relevant part of telemetric measurements, and be robust to the presence of outliers. In average, about 20 to $30 \%$ of the measurements had to be considered as erroneous.

In this experiment, the initial configuration of Minitruck is only known to satisfy $x \in[24 \mathrm{~m}, 25 \mathrm{~m}], y \in$ 
Actual final configuration

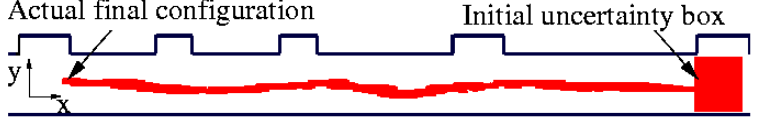

Fig. 4. Tube containing the projection onto the $(X, Y)$-plane of all subpavings evaluated during the tracking of Minitruck by the boundederror state estimator

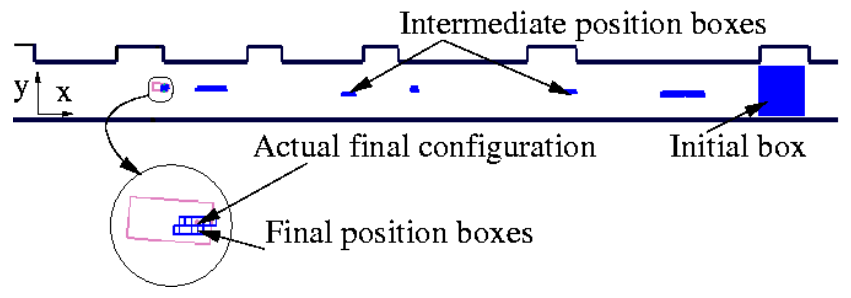

Fig. 5. Projections onto the $(X, Y)$-plane of seven subpavings

$[0.0 \mathrm{~m}, 1.5 \mathrm{~m}]$ and $\theta \in\left[140^{\circ}, 200^{\circ}\right]$.

Fig. 4 represents the projection onto the $(X, Y)$-plane of all subpavings obtained during the tracking of Minitruck, starting from the initial uncertain configuration. All solution boxes were displayed. The path followed by Minitruck is coarsely seen, the width of the tube obtained corresponds to localization uncertainty. Sometimes, the uncertainty is much decreased, due to asymmetry of the environment.

Fig. 5 displays the projection onto the $(X, Y)$-plane of seven estimated configuration subpavings taken during the tracking of Minitruck. Despite of the large amount of erroneous data, a relatively precise localization is provided, often with a precision of about $10 \mathrm{~cm}$.

At the end of this experimentation, the final configuration is well estimated (Table I). The actual configuration is represented on Fig. 5 and contained in the last subpaving provided.

These results show the ability of the bounded-error localization technique to work in a real environment.

\section{B. Global localization with local symmetries}

Starting from a unknown initial configuration, the bounded-error localization method is able to find where Minitruck is, even when local symmetries complicate the task, see Fig. 6.

The initial configurations box is $10 \mathrm{~m}$ long, $1.2 \mathrm{~m}$ large, with an $180^{\circ}$ uncertainty on orientation. The aim is here to show the efficiency of the bounded-error estimator in global localization.

The computing time is much larger than in the first experiment for the first correction step (up to 6 seconds in Fig. 6-1), as the set to be considered is large. After

TABLE I

ACTUAL CONFIGURATION AND BOX CONTAINING THE ESTIMATED CONFIGURATION SUBPAVING PROVIDED BY THE BOUNDED-ERROR LOCALIZATION TECHNIQUE

\begin{tabular}{|c|c|c|}
\hline & Actual configuration & Estimated configuration \\
\hline$X(\mathrm{~cm})$ & 675 & {$[663,688[$} \\
\hline$Y(\mathrm{~cm})$ & 104 & {$[98,107]$} \\
\hline$\theta(\mathrm{deg})$ & 176 & {$[170,177]$} \\
\hline
\end{tabular}

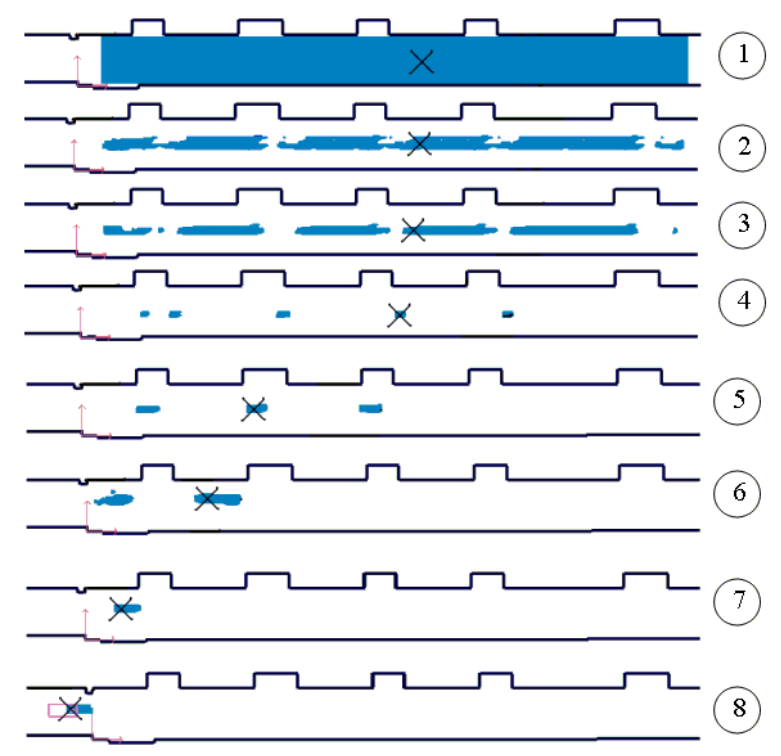

Fig. 6. Bounded-error state estimation with an unknown initial configuration; the cross indicates the actual position. The circled numbers correspond to different instants of time

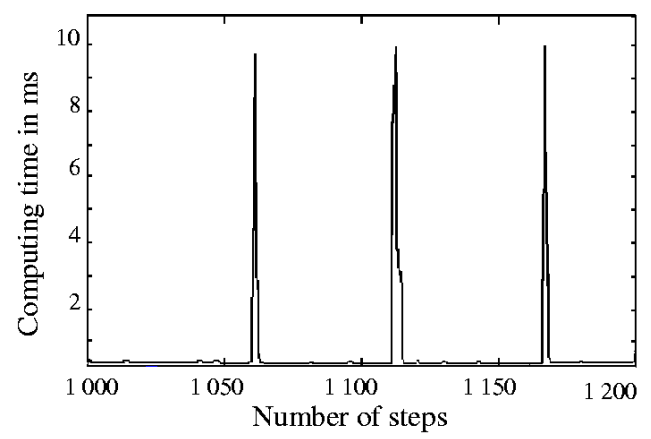

Fig. 7. Computing time

this first correction step, many configurations were deemed inconsistent with the data. A configuration was deemed inconsistent and eliminated as soon as more data had to be considered as outliers than the minimum required to obtain a nonempty configuration set after correction. By deleting the inconsistent configuration the subpaving reduced as in Fig. 6-2 (the cross represents the actual configuration of Minitruck). All boxes in the subpaving were then taken into account for the next prediction and correction steps. After the integration of more data provided by the sonars, Fig. 6-3 shows that the solution subpaving consisted of only seven disconnected components, each of which may contain the actual configuration of Minitruck. Between Fig. 6-4 and Fig. 6-7, the inconsistent configuration subsets were eliminated, and the only surviving configuration set was the one that contained the actual configuration. Most of the configurations were eliminated when Minitruck passed near obstacles corresponding to asymmetries of the map. Fig. 6-8 displays the projection on the $(X, Y)$-plane of the final subpaving, which contains the actual configuration and has a size which is about the same as that obtained in the previous experiment (see Section IV-A).

During four seconds of experimentation between steps 7 
TABLE II

FINAL CONFIGURATION

\begin{tabular}{|c|c|c|c|}
\hline Final config. & Actual & Predicted & Estimated \\
\hline$X(\mathrm{~cm})$ & -4660 & -4234 & {$[-4668,-4650]$} \\
\hline$Y(\mathrm{~cm})$ & 847 & 919 & {$[842,865]$} \\
\hline$\theta(\mathrm{deg})$ & 88 & 47.2 & {$[84,93]$} \\
\hline
\end{tabular}

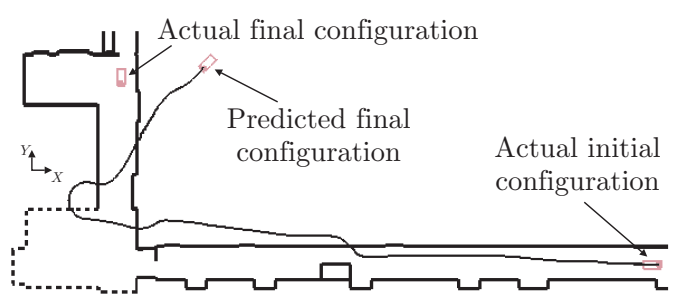

Fig. 8. Consequence of the sole use of odometric data

and 8 , the computing time was measured. At this stage of the experiment, the uncertainty is small. Results are presented on Fig. 7. Correction phases correspond to the peaks. Between each peak, about fifty predictions were performed. For a four-second experimentation the total computing time, on a $2 \mathrm{GHz}$ Pentium IV processor, is less than one second for the bounded-error localization method, which allows real-time localization.

\section{A longer experiment}

During this third experiment, the vehicle followed a $45 \mathrm{~m}$ trajectory. As could be expected, the cumulative odometric errors were even more significant than for the first test, with a discrepancy of more than $4 \mathrm{~m}$ between the predicted and actual positions (see Table II and Fig. 8).

The results provided by the bounded-error method are presented on Fig. 9.

A large room is present at the angle of the map, within which the sonars become unable to provide any meaningful measurements. This is why only prediction was performed in this area. Despite this particularly difficult situation, the state estimator remained capable of computing subpavings that always contained the actual configuration. The final configuration of the vehicle was very accurately computed (see Table II).

\section{CONClusions AND PERSPECTIVES}

In this paper, we presented a robust localization method based on a bounded-error technique and applied it to an actual vehicle. The very noisy data provided by sonars and odometers made the test-case particularly difficult and informative.

The ability of the robust bounded-error estimator to cope with many erroneous data provided by the sonars and to obtain accurate estimates of the vehicle configuration was demonstrated, even when the initial configuration of the vehicle was totally unknown. Moreover, the localization method has shown its ability to deal with many disconnected subsets, corresponding to various localization hypotheses.

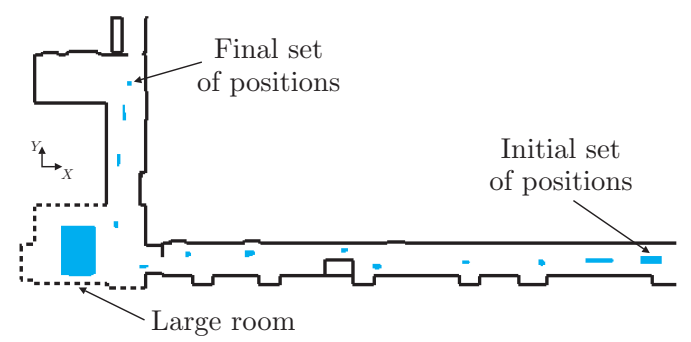

Fig. 9. A few of the sets of positions obtained with the state estimator

Except in the initial steps, when the vehicle configuration is too badly known, the method is compatible with realtime applications. In order to deal with more complex maps and large initial uncertainty, it may be useful to speed up the bounded-error localization. This could easily be achieved by precalculating the sonar simulated measurements as in [4].

Bounded-error localization has now to be compared with other approaches such as Bayesian localization which is presently the most commonly used. This paper did not address the topic of simultaneous localization and mapping (SLAM), where the potential of guaranteed computation based on interval analysis should form the subject of future studies.

\section{REFERENCES}

[1] J. Borenstein, B. Everett, and L. Feng. Navigating Mobile Robots: Systems and Techniques. A. K. Peters Ltd., Wellesley, MA, 1996.

[2] J. L. Crowley. World modeling and position estimation for a mobile robot using ultrasonic ranging. In IEEE International Conference on Robotics and Automation, pages 674-680, Scottsdale, AZ, 1989.

[3] J.J. Leonard and H.F. Durant-Whyte. Mobile robot localization by tracking geometric beacons. IEEE Trans. on Robotics Automation, 7(3):376-382, 1991.

[4] W. Burgard, D. Fox, D. Hennig, and T. Schmidt. Estimating the absolute position of a mobile robot using position probability grids. In Proc. of the 13rd National Conference on Artificial Intelligence, volume 2, pages 896-901, Portland, OR, 1996.

[5] F. Dellaert, D. Fox, W. Burgard, and S. Thrun. Monte Carlo localisation for mobile robots. In Proc. of the IEEE Int. Conf. on Rob. and Autom., pages 1322-1328, Detroit, MI, 1999.

[6] C.K. Chui and G. Chen. Kalman Filtering with Real-Time Applications. Springer Verlag, 1990.

[7] K. Ito and K. Xiong. Gaussian filters for nonlinear filtering problems. In IEEE Transaction on Automatic Control, volume 45, pages 910-927, May 2000.

[8] S. Thrun, D. Fox, W. Burgard, and F. Dellaert. Robust Monte Carlo localization for mobile robots. Art. Int. Jnl, 128(1-2), 2001.

[9] D. Filliat and J.A. Meyer. Global localization and topological map learning for robot navigation. In Hallam et al., editor, Proc. of the Seventh Int. Conf. on Simul. of Adapt. Behav., from Animals to Animats, pages 131-140, The MIT Press, 2002.

[10] M. Kieffer, L. Jaulin, E. Walter, and D. Meizel. Robust autonomous robot localization using interval analysis. Reliable Computing, 6(3):337-362, 2000.

[11] A. Lambert and N. Le Fort-Piat. Safe task planning integrating uncertainties and local maps federation. International Journal of Robotics Research, 19(6):597-611, 2000.

[12] R. E. Moore. Methods and Applications of Interval Analysis. SIAM, Philadelphia, PA, 1979.

[13] H. Ratschek and J. Rokne. Computer Methods for the Range of Functions. Ellis Horwood, Chichester, UK, 1984.

[14] L. Jaulin, M. Kieffer, O. Didrit, and E. Walter. Applied Interval Analysis. Springer Verlag, London, 2001.

[15] L. Jaulin and E. Walter. Set inversion via interval analysis for nonlinear bounded-error estimation. Automatica, 29(4):1053-1064, 1993. 